\title{
Inverse Chill and Nodular Graphite Formation in Synthetic Cast Irons Melted and Cast under Vacuum
}

\author{
By B. K. Dhindaw* and J. D. Verhoeven**
}

\begin{abstract}
Studies done on the solidification characteristics of synthetic cast-irons of various purity levels melted and cast under vacuum varying from $13.3 \mathrm{~Pa}$ to $1.33 \times 10^{-4} \mathrm{~Pa}$ bring out certain significant results. Properly degassed clean cast-irons under conductive heat transfer conditions are prone to inverse chill formation. This happens when growth of eutectic graphite terminates at certain undercoolings and further solidification proceeds by cementite nucleation. The shapes of graphites, as revealed by scanning electron microscopy, at the transition zones between white and gray iron, across the inverse chill zone show how the eutectic graphite coarsens into different forms before its growth is stopped. Nodular graphite forms at high cooling rates and coral graphite forms at low cooling rates in high purity cast-irons only. The conditions like purity level, cooling rates and superheat etc., leading to various nodular graphite densities in such cast irons have been identified. Based on this a theory is proposed explaining the mechanism of formation of nodular graphite in such cast irons involving carbides as nucleating agents. Implications of the above studies in the formation of nodular graphite and inverse chill in commercial cast irons inoculated with magnesium are discussed.
\end{abstract}

(Received August 21, 1982)

Keywords: cast iron, inverse chill, nodular graphite, vacuum melting solidification characteristics, eutectic graphite, inoculation, scanning electron microscopy

\section{Introduction}

Cast Iron from the metallurgical point of view is one of the least understood materials. Of special interest is the formation of the various graphite shapes. A large number of studies have been done so far to understand the solidification behaviour of cast-iron. But the complex metallurgy of cast iron and especially the presence of trace impurities and the sensitivity of the structure to cooling rate has handicapped the clear understanding of the mechanism of graphite precipitation. The formation of graphite spherulites has been observed $^{(1)-(3)}$ in Fe-C-Si alloys when they were melted and cast under vacuum using high purity raw materials. But the results of the studies done so far on formation of graphite spherulities under vacuum using high purity raw materials have not been identical. Apart from it the conditions of formation of coral graphite shapes or rod like graphite shapes are

* Department of Metallurgical Engg., Indian Institute of Technology, Kharagpur (W.B.), India.

** Department of Materials Science and Engineering, Iowa State University, Ames, Iowa, U.S.A. not clearly understood. Some studies refer to the cooling rate as one essential criterion for their formation and some refer to the purity of cast-iron as the essential criterion. Another interesting observation which needs to be taken note of is that 'inverse chill' which is observed in S.G Iron castings has also been found to take place in vacuum melted cast-irons. Thus the solidification mechanism in S.G. Iron and high purity cast-irons melted and cast under vacuum seems to have some similarity. Therefore, there seems to be little doubt about the fact that the future course of work on this subject should definitely be with the aid of 'pure' raw materials and under clean conditions. The present paper describes the results of detailed studies undertaken by the authors on solidification characteristics of $\mathrm{Fe}-\mathrm{C}-\mathrm{Si}$ alloys of varying purity levels melted and cast under clean conditions, with special reference to the formation of different graphite shapes and inverse chill in them.

\section{Experimental}

Basically two different types of charges and vacuum levels were used in these experiments. In one set of experiments the charge consisted 
of electrolytic zone refined iron, semi-conductor grade silicon and spectroscopic grade graphite (referred to as high purity level charge in the text) and in another set of experiments the charge consisted of commercial grade of mild steel, electrode graphite and ferrosilicon (referred to as low purity level charge in the text). The vacuum levels employed in the experiments varied from 13.3 $\mathrm{Pa}$ (referred to as low vacuum level in the text) to $1.33 \times 10^{-4} \mathrm{~Pa}$ (referred to as high vacuum level in the text). The charge in most cases was approximately $30-50 \mathrm{~g}$. The charge in all cases was 3.0 mass $\%$ carbon and $2.4 \mathrm{mass} \%$ silicon, that is, hypoeutectic castirons were only studied. High purity alumina crucibles were used for melting and casting of the alloys. The compositions of resultant melts were nearly identical to the composition of charge. Susceptors were used in various positions around the crucibles. As the susceptors were made of tantalum/graphite, on shutting off or on reducing the power for cooling of melt they acted as heat sinks and led to faster cooling rates along certain directions. This helped in the study of inverse chill formation. The melting was done by placing the charge in alumina crucibles, surrounded by susceptors as indicated above, and by placing this assembly inside a quartz tube which was suitably shielded from the susceptor. The inside of the quartz tube was maintained at the required vacuum level. There was arrangement of back filling the quartz tube with high purity argon gas. Melts were solidified in the crucible itself except in one case when the melt of high purity charge was chill cast inside a water cooled copper mold. All the castings after melting were held under the vacuum for a period of $1.8-2.7 \mathrm{Ks}$ before solidification.

\section{Experimental Results and Discussion}

After solidification the macrostructures and microstructures of polished sections were examined by optical and scanning electron microscopy. The effects of cooling rate, purity level and melt conditions upon these structures were examined. The cooling rates were measured at the onset of the first thermal arrest. Interesting differences in structures occur between the slow and fast cooling rates and also due to variation of purity levels and melt conditions in these cast-irons. At slow cooling rates and high purity levels of charge melted and cast under high vacuum, inverse chill (which is occassionally observed in S.G. Iron castings) was formed in the castings as shown in Fig. 1. It consisted of white iron island inside an outer structure consisting of ferrite and coral graphite. Sometimes 1 or 2 isolated nodules were observed in the central white iron region. The coral graphitic structure is confirmed as seen from the scanning electron micrograph taken from gray iron portion after deep etching the matrix (Fig. 2). The graphite shows the branched rod like structure, typical of coral graphite. On

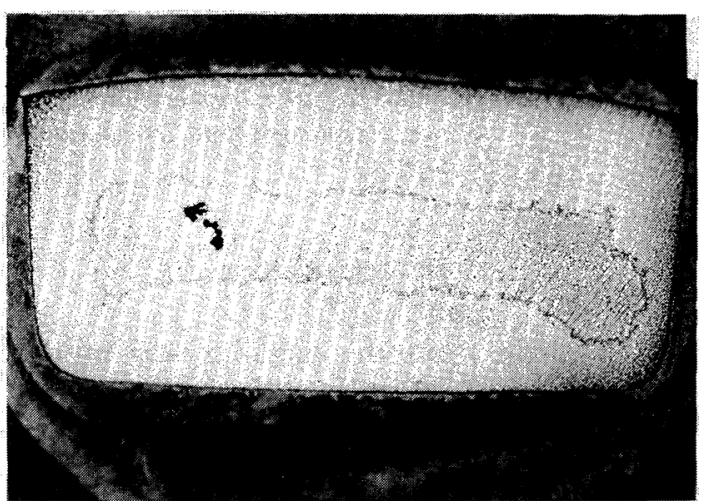

Fig. 1 Macrograph of slowly cooled castings made from very high purity charge, melted and cast under high vacuum showing inverse chill in the center.

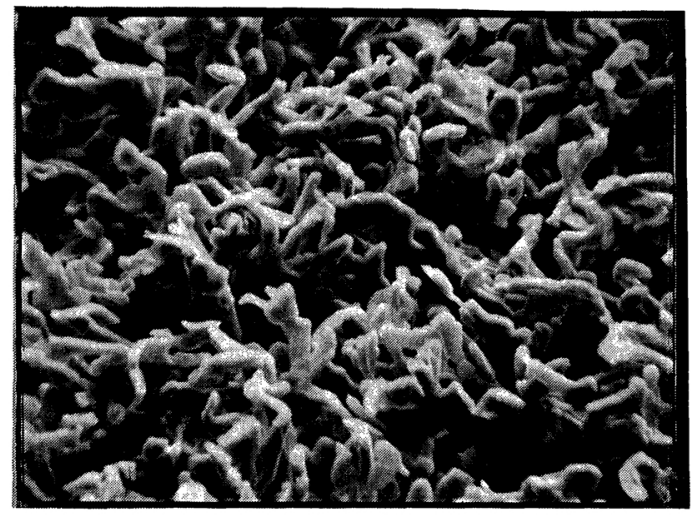

Fig. 2 Scanning electron micrograph showing coral graphite structure in the gray portion of the castings shown in Fig. 1 deep etched with Nital $\times 1200 \times 2 / 3$. 
further increase of the cooling rate to the order of $2 \mathrm{~K} / \mathrm{s}$ or more, the cast-iron of these high purity levels melted under high vacuum showed a general white iron matrix in the centre of which massive graphite had precipitated, and it was dendritic in nature or the inverse chill got inversed. Figure 3 shows the typical macrograph of such a structure. The inner region of the casting solidified as white cast iron contained many graphite nodules (Fig. 4). Deep etching experiments clearly showed these were true nodules. The structure of high purity cast iron melted under high vacuum and chill cast in water cooled copper mold was completely white and contained no nodules. When the low purity level charge and the low vacuum levels were used at cooling rates as high as upto $4.17 \mathrm{~K} / \mathrm{s}$, the structure consisted of ferrite and eutectic graphite, and its deep etched structure

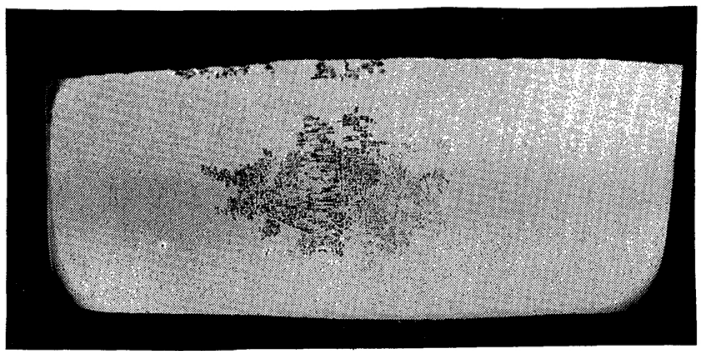

Fig. 3 Macrograph showing the massive compact graphite in the center of fast cooled castings made from high purity charge, melted and cast under high vacuum.

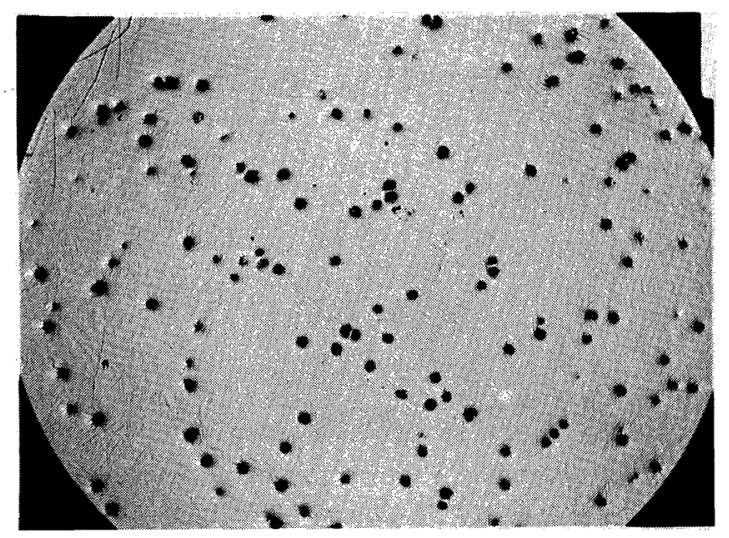

Fig. 4 Micrograph showing number of graphite nodules precipitated in the matrix of the casting shown in Fig. 3. Unetched, $\times 38 \times 3 / 5$. is shown in Fig. 5. The graphite shape is little different than normal type D graphite shape. However, when the high purity charge was melted and cast under low vacuum, inverse chill could be observed upto cooling rates of the order of $3.3 \mathrm{~K} / \mathrm{s}$. The typical macrostructure was here same as of in Fig. 1 i.e. about $40 \%$ structure was white iron in the centre, rest grey. However there were some major differences. The outer regions consisted of ferrite and eutectic graphite, with a structure akin to coral graphite. The centre region of the casting had majority of structure as white iron, but there were two regions where massive graphite of dendritic nature had precipitated. The white iron region showed large nodular graphite precipitation. On deep etching it was revealed that some nodules in this case were slightly degenerated. Thus the structure here consists of both inverse chill and inverse of inverse chill.

In all the above cases the susceptor completely surrounded the crucible. In the case of the low purity charge under low vacuum, only when the suscepter was placed at the bottom of the crucible and the sides were insulated, inverse chill was obtained near the bottom of the casting, at the high cooling rates, otherwise nearly fully grey iron structure was observed till high cooling rates.

The gray white transitions zones were examined in all the above cases carefully, as fascinating structures were obtained here. Figure 6, shows the micrograph showing the transition zone in case of high purity cast-irons melted

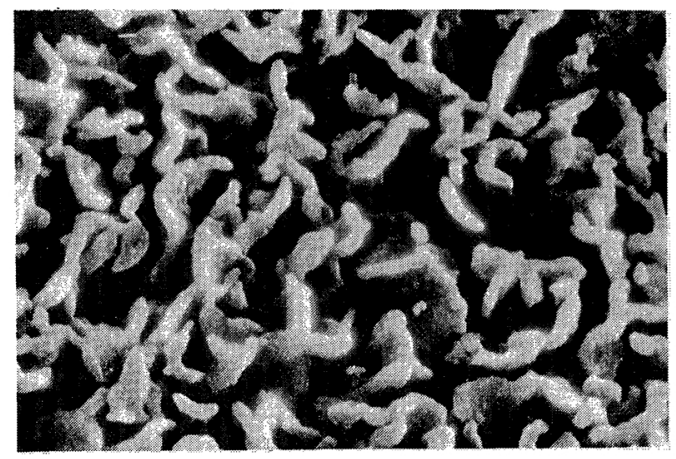

Fig. 5 Scanning electron micrograph showing the morphology of eutectic graphite in the castings made from low purity cast-irons melted under low vacuum. Deep etched Nital, $\times 3300 \times 2 / 3$. 


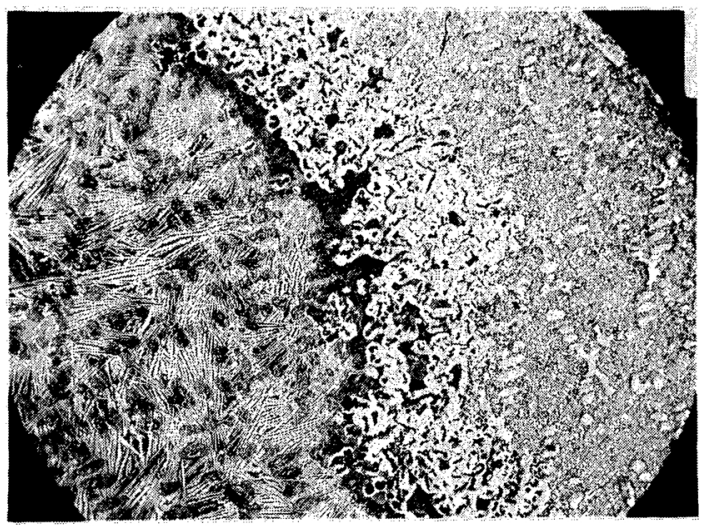

Fig. 6 Micrograph showing the structure in the transition zone between the white and gray regions of the casting shown in Fig. 1. Etched Nital, $\times 38 \times 3 / 5$.

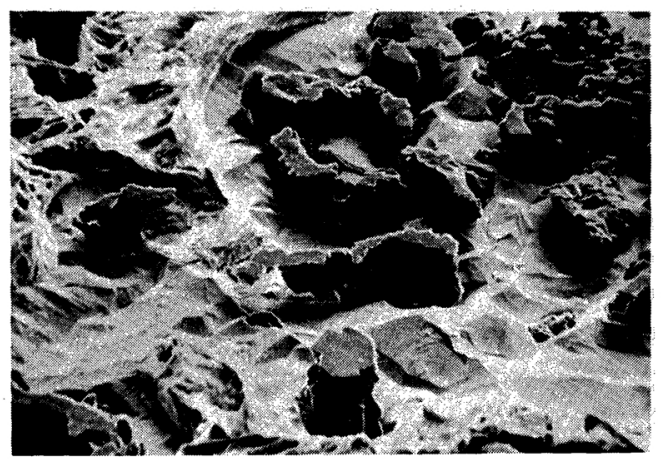

Fig. 7 Scanning electron micrograph showing the different graphite shapes as observed in the transition zone, shown in Fig. 6. Deep etched with Nital, $\times 450 \times 2 / 3$.

under high vacuum. The transition zone shows gradual thickening of coral graphite to thick graphite shapes which on deep etching show rod like morphology of graphite. Figure 7, shows the deep etched scanning electron micrograph of this region. The high purity cast irons melted under low vacuum but at high cooling rates showed a rather narrow transition zone, where thickening of coral graphite was not to that extent. Figure 8 shows the scanning electron micrograph of the deep etched structure from this zone. It can clearly be seen that eutectic graphites growth suddenly terminates at the transition zone (of course after little thickening) and then white iron region starts. In case of impure irons at low vacuum, the transition was still more sudden, as seen from micrograph

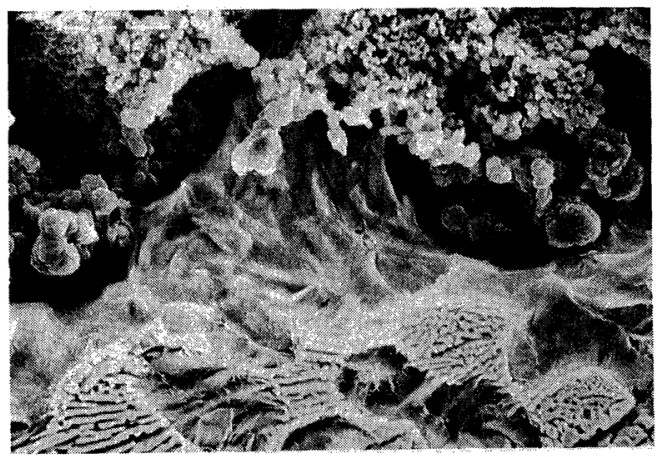

Fig. 8 Scanning electron micrograph of the transition zone of high purity cast-irons melted under low vacuum, showing lesser thickening of the eutectic graphite on termination of growth. Deep etched with Nital, $\times 480 \times 2 / 3$.

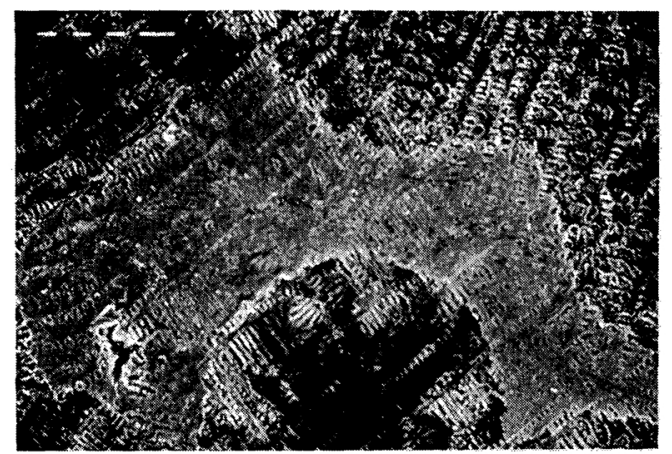

Fig.9 Scanning electron micrograph showing the transition zone across the inverse chill in low purity cast irons melted under low vacuum. Deep etched Nital, $\times 42 \times 2 / 3$.

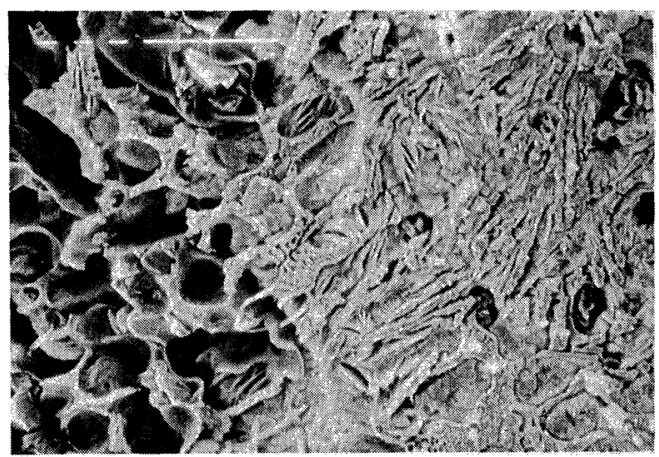

Fig. 10 Scanning electron micrograph showing the transition zone between the central massive graphite region and white iron region in high purity cast irons melted under low vacuum. Deep etched Nital, $\times 172 \times 2 / 3$ (left, massive graphite, right, white iron with nodules). 
shown in Fig. 9. The transition zone from white iron to central massive graphite portion was rather abrupt, and it was continuous with the austenite dendrites which extended throughout this region. Figure 10 shows a typical micrographs across this region. This pattern was identical in all castings. When this charge consisted of very high purity zone refined iron, semiconductor grade silicon and spectroscopic grade graphite and it was melted and cast under high vacuum at cooling rates upto $3.3 \mathrm{~K} / \mathrm{s}$, the structure showed white iron matrix with central massive graphite regions. However, the basic difference between this structure and those under similar conditions produced in high purity melts under high/low vacuum was that there were very few graphite nodules precipitated in the white iron region. In fact there were only one or two nodules in the whole region. However, when such irons prior to solidification under the above conditions of melting and holding were inoculated by commercial ferrosilicon, they did contain a large number of grains of nodular graphite in white iron regions, with nodules being slightly degenerated. The condition which increased the nodular count in the high purity cast irons melted and cast under high vacuum was holding it at high temperatures (around $1870 \mathrm{~K}$ ) prior to solidification after vacuum refining it at $1723 \mathrm{~K}$, and the condition which drastically reduced the nodular count in the above cast irons was holding it at low temperatures (around $1550 \mathrm{~K}$ ) prior to solidification after vacuum refining it at $1723 \mathrm{~K}$. However, in the latter case inoculation prior to solidification produced a large number of nodular graphite grains. Table 1 lists analysis of the typical cast irons produced from zone refined iron (referred to a very high purity iron) and electrolytic iron (referred to a high purity iron) as obtained by the spark source mass spectroscopic analysis. It is noticed from the analysis that Al-pick-up from the crucible takes place upto the extent of $60 \mathrm{ppm}$ (the analysis was done with the help of highly accurate gravimetric methods). To check whether there is an overall effect of $\mathrm{Al}$ in this range on the structure, control melt was taken using a thoria crucible with a thoria thermocouple protector. The resultant micro-
Table 1 Typical analysis of cast-irons melted and cast under high vacuum in the present studies (all numbers ppm by mass).

\begin{tabular}{lcl}
\hline \hline Element & $\begin{array}{c}\text { Cast irons made } \\
\text { using electrolytic } \\
\text { iron }\end{array}$ & $\begin{array}{c}\text { Cast irons made } \\
\text { using zone refined } \\
\text { iron }\end{array}$ \\
\hline $\mathrm{Li}$ & 0.001 & 0.0004 \\
$\mathrm{Be}$ & 0.003 & 0.002 \\
$\mathrm{~B}$ & 0.2 & 0.08 \\
$\mathrm{Mg}$ & 0.4 & 0.9 \\
$\mathrm{P}$ & 1.7 & 0.1 \\
$\mathrm{~S}$ & 0.3 & 0.2 \\
$\mathrm{Ca}$ & 0.1 & 0.1 \\
$\mathrm{Ti}$ & 0.4 & 0.07 \\
$\mathrm{Cr}$ & 0.9 & 0.6 \\
$\mathrm{Mn}$ & 0.6 & 0.4 \\
$\mathrm{Co}$ & 5 & 0.4 \\
$\mathrm{Ni}$ & 4 & 2 \\
$\mathrm{Cu}$ & 0.1 & 0.1 \\
$\mathrm{Ge}$ & 8 & 0.4 \\
$\mathrm{~Pb}$ & 1.5 & 0.3 \\
$\mathrm{O}$ & 10 & 3 \\
$\mathrm{H}$ & 1 & 1 \\
$\mathrm{~N}$ & 1 & 1 \\
$\mathrm{Al}$ & 62 & 64 \\
$\mathrm{C}$ & 3.03 mass $\%$ & 3.07 mass $\%$ \\
$\mathrm{Si}$ & 2.51 mass \% & 2.50 mass $\%$ \\
\hline \hline $\mathrm{Al}$ & & \\
\hline
\end{tabular}

All analysis except $\mathrm{Al}, \mathrm{C}$ and $\mathrm{Si}$ by spark source mass spectrophotometry. Gases, $\mathrm{O}, \mathrm{H}, \mathrm{N}$ by vacuum fusion technique.

structure was identical to that obtained using the alumina crucible at the identical cooling rate. The resultant $\mathrm{Al}$ in melt when thoria crucible was used was around $20 \mathrm{ppm}$. In order to confirm that there was no overall segregation of $\mathrm{Si}, \mathrm{X}$-ray microprobe analysis traces were taken across a transition zone of high purity cast-iron melted under low vacuum. The result presented in Fig. 11 shows no such segregation.

In a series of papers Doi et al. ${ }^{(4)-(6)}$ report on experiments similar to the present work in which $50 \mathrm{~g}$ samples of $\mathrm{Fe}-\mathrm{C}-\mathrm{Si}$ alloys prepared from electrolytic iron were vacuum melted in $\mathrm{Al}_{2} \mathrm{O}_{3}$ crucibles. They obtained inverse chill structures with the fraction of grey iron (presumably coral graphite or eutectic graphite) decreasing with increasing cooling rates. They also report that the formation of graphite nodules increases with both melt temperature and cooling rate, but they apparently observed nodular graphite only with hypereutectic alloys $^{(5)}$. They do not report on the chemical 


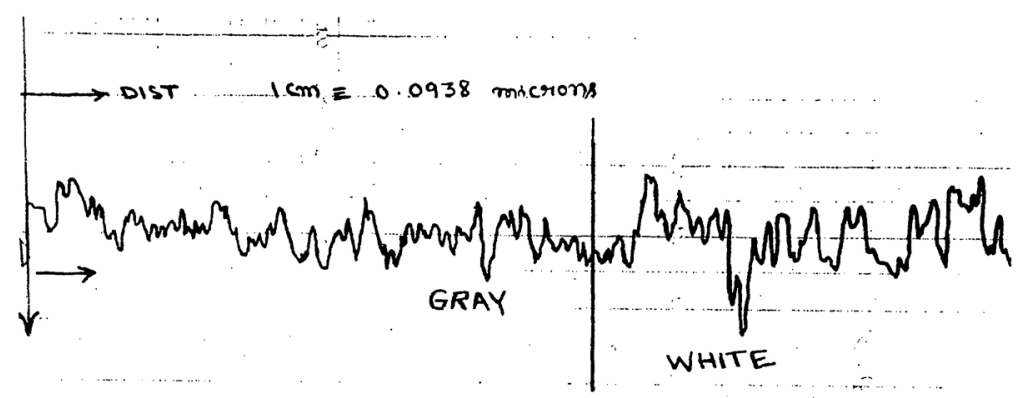

Fig. $11 \mathrm{X}$-ray microprobe trace across the transition zone between gray and white iron region of the casting shown in Fig. 8.

analysis for their melts.

Honma and Minato $^{(7)}$ report a vacuum melting of synthetic $\mathrm{Fe}-\mathrm{C}-\mathrm{Si}$ alloys in which they find nodular graphite formation in a white iron matrix. They have done their experiments with low purity iron. Maruyama ${ }^{(8)-(10)}$ has shown that inoculation of vacuum degassed synthetic $\mathrm{Fe}-\mathrm{C}-\mathrm{Si}$ alloys with ferrosilicon inoculant produces nodular graphite structures in portions of the castings.

Chernoval and Khusnutdinov ${ }^{(11)}$ have studied $20 \mathrm{~g}$ samples of synthetic hypereutectic irons vacuum degassed in $\mathrm{Al}_{2} \mathrm{O}_{3}$ crucibles at $1773 \mathrm{~K}$. They find nodular graphite formation only at higher cooling rates and low sulpher contents, rates of $2.0 \mathrm{~K} / \mathrm{s}$ and above and $\mathrm{S}$ $10 \mathrm{ppm}$. In a similar study by Akhmatov et $a l^{(12)}$ on high purity hypereutectic irons the same results were obtained, i.e., nodules were favoured at high cooling rates and high purities.

$\mathrm{Lux}^{(13)}$ refers to unpublished research on vacuum melted pure $\mathrm{Fe}-\mathrm{C}-\mathrm{Si}$ alloys which supports the present findings. Nodular graphite was reported to form at high cooling rates, while slow cooling rates producing coral graphite morphology. Some of the results in the present paper have been discussed earlier ${ }^{(14)}$. However, a combined discussion on all the results presented here is given below.

\section{Inverse Chill}

In most cases when inverse chill occurs, the cooling curves show the two stage eutectic arrest, Fig. 12. The cooling curves indicate that the coral graphite nucleates within the

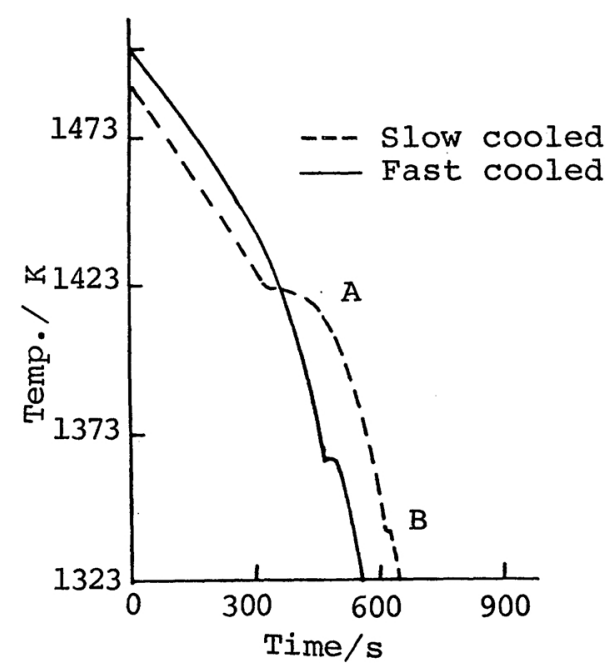

Fig. 12 Typical cooling curves for slow cooled casting shown in Fig. 1 (having inverse chill) and fast cooled casting shown in Fig. 3 (completely chilled).

interdendritic liquid at around eutectic arrest point $1423 \mathrm{~K}$ and probably stops growing at time $\mathrm{A}$, because here a significant increase in the cooling rate is observed. The white iron did not nucleate until time B. Hence, during this interval A-B i.e. between the two arrest points the eutectic graphite/iron growth front was not able to continue the growth and fill the center of the casting. Hence, as the eutectic graphite front grows towards the center of the casting, the casting temperature drops and the undercooling increases. When the undercooling reaches point $\mathrm{A}$, the growth rate of the eutectic graphite morphology has dropped sharply and its growth essentially comes to a halt. The remaining interdendritic melt, trapped in the casting center, then cools 
without further transformation until white iron uncleates at around B (Fig. 12). These results raise two interesting questions.

I. Why does the coral graphite growth terminate as the undercooling increases?

II. Why does not significant additional nucleation of graphite occur within the center liquid portion as it cools between points $\mathrm{A}$ and $\mathrm{B}$ ?

First Question I will be considered and Question II will be deferred to later. Three possible reasons come to mind as to why the coral graphite growth terminates.

a) Perhaps a composition change has occurred at the coral growth front which halts the growth.

b) Perhaps at the increased undercoolings the branching mechanism for the cooperative growth of graphite and austenite becomes less effective, causing the growth to stop.

c) Perhaps graphite nucleates in the liquid immediately in front of the coral growth front and stops its growth.

The micrographs of Figs. 6, 7 and 8 show that at the front where the growth of the coral graphite stopped, significant coarsening of the graphite has occurred. Hence, it seems unlikely that mechanism ' $c$ ' is correct. If mechanism ' $a$ ' is responsible, the composition change must involve either impurity elements or the three ternary elements. It seems unlikely that impurity segregation is responsible, because the volume of the chill region was essentially the same for both electrolytic and zone refined irons under indentical melting conditions. As discussed by Lux and $\mathrm{Kurz}^{(15)}$, coral graphite forms by essentially a cooperative growth between austenite and graphite into the liquid iron. Although there must be a fluctuating diffusion field in the liquid at the growth front due to the irregular graphite morphology, one expects that diffusion is complete in the lateral direction and no long range diffusion field develops normal to the growth front, so that the front moves under near steady state conditions. Hence, one would not expect significant changes in the ternary $\mathrm{Fe}-\mathrm{C}-\mathrm{Si}$ composition as the growth front migrates toward the casting centre.
Little is known about the cooperative growth mechanism for coral graphite and austenite. Perhaps at increased undercooling the branching mechanism required for cooperative growth of the graphite breaks down due either to changes in the atomic attachment kinetics or to a very small impurity buildup. Then, when the cooperative growth mechanism stops, randomly selected graphite centers coarsen and the graphite growth halts or becomes extremely small, because the existing degree of undercooling does not match the kinetic requirements for a continued growth of this new graphite morphology. Hence, it seems most probable that mechanism ' $b$ ' or perhaps a combination of ' $b$ ' and ' $a$ ' is responsible for the termination of the coral growth front. The idea that inverse chill forms because the growth of the coral graphite is halted at increased undercoolings may also be applicable to commercial cast irons, because it is found ${ }^{(16)}$ that inverse chill occurs most often in irons containing undercooled graphite. Perhaps the growth of undercooled graphite also is halted at increased undercoolings thereby allowing the remaining liquid to solidify white. However, the eutectic graphite cannot coarsen to that extent here as in case of coral graphite when the growth is halted as observed in case of impure irons in the present structures (Fig. 8).

Initial nucleation of the coral graphite at the periphery of the casting having inverse chill, however, seems to be a function of purity levels of the melt with respect to the extent of vacuum degassing, as in melt of high purity charge melted and cast under low vacuum peripheral eutectic graphite/ferrite structures was obtained even at high cooling rates. The formation of inverse chill zones in directions of placement of susceptor/chill is due possibly to the favourable conditions of undercooling created in these regions caused by favourable heat transfer conditions. Such formation of inverse chill is a function of both purity levels and heat transfer conditions.

\section{Nodular Graphite Formation}

The present work brings out some characteristic features about nodular graphite 
formation in samples of cast-irons considered. They can be summarized as:

1. Nodular graphite forms neither in high purity melts (using electrolytic iron) nor in very high purity melts (using zone refined iron) at low cooling rates. The coral graphite morphology always forms.

2. Nodular graphite does form in the high purity melts at the high cooling rates, when cooled from high temperatures 1723-1893 K, but does not form if (a) very high purity zone refined iron is used or (b) the melt is solidified from low temperatures $1553 \mathrm{~K}$.

3. The melts cooled from higher temperature $1893 \mathrm{~K}$ have higher nodular density than the melts cooled from $1723 \mathrm{~K}$.

4. Addition of inoculant produces nodular graphite under the conditions 2(a) and 2(b), when they were not formed.

Several papers ${ }^{(13)(17)-(23)}$ have emphasised the role which inoculants play in the formation of nodules.

There are strong arguments that the active nucleant for graphite might be a carbide ${ }^{(22)}$ or a sulfide ${ }^{(18)(20)}$. However, the support to such ideas based on conclusive experimental evidence is lacking. In our case the possibility of sulfide being an active nucleating agent is not favoured, as nodular graphite was only observed in high purity cast irons in which sulphur is expected to be very low.

In the present case it is proposed that carbides are involved as nucleating agents. When we melt the cast irons at very low sulpher and oxygen levels some elements present in the system react with carbon and form carbides. The formation of carbides would be favoured by:

(1) Low oxygen level in the melt i.e. good degassing, by holding under vacuum at high temperatures. This is due to thermodynamic reasons.

(2) High temperatures i.e. reaction being favoured kinetically, but thermodynamically probably not favoured for all carbides.

(3) Good agitation in the baths (this happens when the cast-irons in the present work, under high vacuum, were heated to high temperatures $1893 \mathrm{~K}$ ) as heavy degassing occurs. Again this is due to the kinetic reasons as the metallic atoms can interact much better with carbon.
(4) Last but not the least, presence of carbide forming elements i.e. extremely pure melts will not have significant carbide formation unless inoculated. Now when we cool the melts, some of these carbides would dissociate ${ }^{(24)}$ because they become thermodynamically less stable and nascent graphite would percipitate on their surface. This graphite would favour epitaxial growth of graphite which would lead to nodular graphite precipitation.

Some conditions which would favour the dissociation of carbides to produce graphite nuclei are:

(1) Moderately fast cooling rates. Very fast cooling rates (as in case of chill casting) in the copper mold would not cause sufficient break down of carbides. Slow cooling rates would cause redissolution of carbon precipitated into the melt.

(2) Cooling after holding the melt at higher temperatures, as this would give a large free energy change favouring the breakdown of the carbides.

(3) Inoculation of the melt prior to solidification as this would raise the activity of carbon in the melt, and would, therefore, hinder redissolution of graphite precipitated as a result of carbide dissociation into the melt and in case of very high purity melts would carry nucleating elements. The experimental results in the present studies support the above postulated mechanism. It is to be remembered that the amount of carbide forming elements required need not be high. The presence in ppm level is enough to cause the above mechanism to be operative. However, nucleation of coral graphite at the slow cooling rates does not seems to be difficult, as the coral graphite nucleates around eutectic temperatures irrespective of the purity of cast irons. However, one might expect the coral graphite formation to be less sensitive to nucleation rate than nodular formation because of the different growth modes involved. In order to transform a given volume of liquid to the coral graphite form, one needs, in principle, only one nucleation event, whereas to transform the volume to nodular graphite, one requires one nucleation event for each nodule.

The mechanism postulated here for the 
Table $2^{(24)}$

Carbides which become less stable at lower temperatures (expected to help in nodular graphite formation

\begin{tabular}{ll}
\hline $\mathrm{Co}_{2} \mathrm{C}, \mathrm{Co}_{3} \mathrm{C}, \mathrm{CoC}_{2}$ & $\mathrm{NbC}, \mathrm{TaC}, \mathrm{Al}_{4} \mathrm{C}_{3}$ \\
$\mathrm{Mn}_{3} \mathrm{C}, \mathrm{Mn}_{7} \mathrm{C}_{3}$ & $\mathrm{SiC}, \mathrm{TiC}, \mathrm{ZrC}$ \\
$\mathrm{Cr}_{23} \mathrm{C}_{6}, \mathrm{Cr}_{7} \mathrm{C}_{3}, \mathrm{Cr}_{3} \mathrm{C}_{2}$ & $\mathrm{ThC}, \mathrm{VC}$ \\
$\mathrm{UC}$ & \\
\hline \hline
\end{tabular}

nodular graphite formation might have some applicability to commercial nodular irons where sulpher is deliberately low. The function of the nodulizer $\mathrm{Mg}$ would be both to act as a scavenger for sulpher and oxygen thereby promoting the nodular morphology and also to form a moderately stable carbide to promote nucleation. Elements which should be excellent scavengers and yet do not promote nodular formation, such as $\mathrm{Al}$ and $\mathrm{Ti}$, could be rationalized to be ineffective because of their very stable carbides. Table 2 gives the relative stability of carbides of some elements which support the above proposition.

The mechanism based on the gas bubble theory does not seem to be operative here, as the gas content in very high purity cast irons melts and high purity cast-iron melts was more or less identical, but whereas the former had very few nodules, the latter had a large nodule count. Also cooling high purity melts under vacuum and under argon did not show any significant difference in nodule count when rest of the conditions were maintained constants, and this should not be the case, as gas bubbles stability in the above two cases would be quite different.

\section{Conclusions}

(1) Inverse chill is observed, only when sufficient supercooling is induced in the castiron melts under suitable heat-transfer conditions and when the growth of eutectic graphite can be brought to halt.

(2) Formation of inverse chill is effected by cleanliness of the melt induced by degassing under vacuum, as less degassed melts of high purity cast-irons under low vacuum show in- verse chill even at high cooling rates, but same melts degassed heavily under high vacuum are completely chilled.

(3) The inverse of inverse chill forms only in fast cooled high purity cast-iron melts in the white iron regions, possibly due to slowing down of cooling there as a result of heat liberated due to white iron formation.

(4) Nodular graphite forms only in high purity cast-irons melts and not in low purity melts where at identical cooling rates eutectic graphite precipitates.

(5) The impurities causing the nodular graphite nucleation in high purity cast-iron is present at levels of the order of ppm.

(6) The results obtained in the present work support the theory that certain types of carbides act as nucleating agent for nodular graphite formation, by decomposition and liberating nascent graphite which is the active nucleant.

\section{Acknowledgement}

Major portion of the work reported here was done at Ames Laboratory-USDOE, (Iowa State University, Ames, Iowa, U.S.A.), under contract No. W-7405-eng-82.

\section{REFERENCES}

(1) J. Kaverian, H. F. Taylor and J. Wulff: Amer. Found., 23 (1963), 85.

(2) J. P. Sadocha and J. E. Gruzleski: The Metallurgy of Cast Iron, Georgi Pub. Co., St. Saphorin, Switzerland, (1975), 443.

(3) P. M. Thomas and J. E. Gruzleski: J. Iron Steel Inst. 221 (1973), 426.

(4) M. Doi, S. Nishi and Y. Ueda: Imono, 42 (1970), $535,544$.

(5) M. Doi, S. Nishi and Y. Ueda: Imono, 43 (1971), 366.

(6) M. Doi, S. Nishi and Y. Ueda: Imono, 42 (1973), 871.

(7) M. Homma and A. Minato: Imono, 31 (1959), 1064.

(8) M. Maruyama: Imono, 33 (1961), 266.

(9) M. Maruyama and H. Chida: Imono, 30 (1958), 880.

(10) M. Maruyama: 30th International Foundary Congress, Prague, 1963, p. 177.

(11) A. V. Chernovol and G. D. Khusnutdinov: Russ. Cast. Prod., (1969), 11, 523.

(12) Yu. S. Akhmatov, Yu. N. Taran, K. P. Bunin, A. G. Lisnyak and N. Ya. Zaspenko: Russ. Cast. Prod., (1976), 1, 3.

(13) B. Lux: Giesserei Forschung, 22 (1970), 65, 161. English Transl.: AFS Cast Met. Res. J., 8 (1972), 
$25,49$.

(14) B. Dhindaw and J. D. Verhoeven: Met. Trans., 11 A (1980), 1049.

(15) B. Lux and W. Kurz: The Solidification of Metals, ISI Publ. 110, The Iron and Steel Inst., London, England, (1968), p. 193.

(16) H. Morrogh and W. J. Williams: J. Iron Steel Inst., 155 (1947), 349.

(17) A Moore: AFS Trans. 81 (1973), 268.

(18) J. F. Wallace: AFS Trans.: 83 (1975), 75.

(19) M. H. Jacobs, T. J. Law, D. A. Melford and M. J. Stowell: Metals Tech., 1 (1974), 490.
(20) M. J. Lalich and J. R. Hitchings: AFS Trans., 84 (1976), 653.

(21) R. Hummer: The Metallurgy of Cast Iron, Georgi Pub. Co., St. Sophorin, Switzerland, (1975), p. 147.

(22) B. Lux: Recent Research on Cast Iron, Gordon and Breach, New York, (1968), p. 241.

(23) V. H. Patterson and M. J. Lalich: AFS Trans., 86 (1978), 33.

(24) T. Rosenquist: Principles of Extractive Metallurgy, McGraw Hill Book Co., New York, (1974), p. 527. 